\title{
Publisher's Note: Quantum resonant activation [Phys. Rev. E 95, 042104 (2017)]
}

\author{
Luca Magazzù, Peter Hänggi, Bernardo Spagnolo, and Davide Valenti
}

(Q) (Received 14 May 2018; published 18 May 2018)

DOI: 10.1103/PhysRevE.97.059903

This paper was published online on 4 April 2017 with an error in Eq. (A2). Equation (A2) should read as

$$
\dot{P}_{L}(t)=-W^{+}(t) P_{L}(t)
$$

The equation has been corrected as of 4 May 2018. The equation is incorrect in the printed version of the journal. 